\title{
Cycles in symmetric sequence processing
}

\author{
Fernando L. Metz and Walter K. Theumann \\ Instituto de Física, Universidade Federal do Rio Grande do Sul, Caixa Postal 15051, 91501-970 \\ Porto Alegre, Brazil
}

\begin{abstract}
The competition between pattern reconstruction and sequence processing is studied here in an exactly solvable feed-forward layered neural network model of binary units and patterns near saturation. We show results for both symmetric and asymmetric sequence processing, either one competing with pattern reconstruction represented by a Hebbian interaction, in order to compare these two kinds of sequence processing. Phase diagrams of stationary states are obtained and a new phase of cycles of period two is found for a weak Hebbian term in the case of symmetric sequence processing, independently of the number of condensed patterns $c$ which have macroscopic overlaps with the states of the network. In contrast, the stability of these cycles depends strongly on $c$. These results are in contrast with those for the competition between a Hebbian interaction and an asymmetric sequence processing interaction [1], in which the period of the cycles is $c$ and the stability of these solutions does not depend on $c$. The dynamics of the macroscopic overlaps in the stationary cyclic phase is analyzed in both models.
\end{abstract}

Keywords: Layered neural networks; sequence processing; phase diagrams

PACS: 87.10.+e, 64.60.Cn, 07.05.Mh

\section{INTRODUCTION}

Models of associative memory - the ability of a network to retrieve stored information (patterns) using as clues corrupted sets of this information - concentrate mostly on systems with symmetric couplings, i. e., the connection between two neurons satisfy $J_{i j}=J_{j i}(i \neq j)$. In this case the system will always evolve to an equilibrium configuration which is a local minimum of an energy function, and the tools of statistical mechanics can be applied. The simplest way to endow the network with properties of associative memory is to choose a Hebbian interaction between a pair of neurons $i$ and $j$, given by $J_{H}=N^{-1} \sum_{\mu} \xi_{i}^{\mu} \xi_{j}^{\mu}$, the dependence on the units being implicit, where $N$ is the total number of units and $\xi_{i}^{\mu}$ represents the component $i$ of the stored pattern $\mu$. The most representative model with these features is the Hopfield model [2].

Instead of the retrieval of individual patterns statically, another interesting task for a neural network is to reproduce a sequence of stored patterns. The simplest way to induce transitions between patterns is by means of a modified Hebbian learning rule $J_{A}=N^{-1} \sum_{\mu} \xi_{i}^{\mu+1} \xi_{j}^{\mu}$, in asymmetric sequence processing (ASP) in which a pattern is connected to the next one in the sequence, combined with a parallel execution of the neural dynamics. The synaptic matrix in this case is asymmetric and equilibrium statistical mechanics cannot be applied. A dynamical procedure must be used in that case to find the stationary states of the network. Asymmetric interactions may, eventually, give rise to cyclic or to chaotic behavior.

One of the first models for temporal association between patterns by recalling time

CP887, Cooperative Behavior in Neural Systems: Ninth Granada Lectures edited by J. Marro, P. L. Garrido, and J. J. Torres

(C) 2007 American Institute of Physics 978-0-7354-0390-1/07/\$23.00 
sequences and cycles was introduced by Sompolinsky and Kanter [3]. It is a recurrent neural network of binary units that stores a finite number of patterns in which the synapse $J$ between a pair of neurons is composed of a Hebbian term $J_{H}$, which tends to stabilize the network in a pattern, and an ASP term $J_{A}$, which induces transitions between the patterns. The resulting competitive interaction between a pair of neurons is given by $J=J_{H}+v J_{A}(0 \leq v<\infty)$. In addition, a slow dynamic response was introduced in $J_{A}$ as a finite time delay of response of a neuron at one end of a synapse to a signal at the other end. Simulations performed in that work showed that, when $v$ is sufficiently large, the network stays in a pattern for a finite period of time after which a transition is made to the next pattern in the sequence. Much progress has been done on purely ASP and its competition with a Hebbian interaction. The model without time delays in the synapses was studied analytically [4] and the effects of the correlation between the stored patterns on the stationary states of the network was also analyzed [5]. Complete phase diagrams and some results on the transient dynamics, including the presence of cycles of period $c$ with stability properties that do not depend on $c$, were presented in these works.

More recently, there has been a revival of interest in the behavior of these models for the storage of a macroscopic number of patterns. Düring et al. [6] analyzed the properties of the stationary states (the storage capacity and the phase diagram) of a recurrent network for purely ASP, without pattern reconstruction, and a solution for the transient dynamics of the model was recently discussed [7]. The effects of stochastic noise on the model [4] were only recently analyzed in a feed-forward neural network architecture [1], in which an exact solution for the dynamics and complete phase diagrams of stationary states were obtained. The phase of cyclic solutions are not qualitatively affected by the presence of stochastic noise and the properties remain the same as in the finite loading case. A region of non-stationary quasi-periodic states was also found in a range of parameters where $J_{H} / J_{A} \approx 1$.

Another interesting way to associate patterns sequentially is by the introduction of another sequence composed by the same set of patterns in opposite order. Thus, for symmetric sequence processing (SSP), $J_{S}=N^{-1}\left(\sum_{\mu} \xi_{i}^{\mu+1} \xi_{j}^{\mu}+\sum_{\mu} \xi_{i}^{\mu-1} \xi_{j}^{\mu}\right)$ and a given pattern is connected simultaneously to the next pattern and to the previous one in the sequence with the same weights, in a way that it is more difficult to know a priori the effects of on the dynamics of the network.

This kind of sequence processing was first introduced in order to explain experimental findings in the cortex of monkeys $[8,9,10]$. In one of the experiments, correlations in the internal representations of stimuli, chosen to be uncorrelated, were observed when the stimuli were presented during training in a fixed sequence. To account for this temporalspatial conversion, Griniasty et al. [11] proposed a model in which the synaptic matrix is composed of a Hebbian term $J_{H}$ in competition with SSP. They obtained activity patterns (fixed-point attractors), each one due to the presentation of one of the uncorrelated stimuli (patterns), that are significantly correlated with several patterns in the learning sequence up to a finite distance from the stimulated pattern. This fact seems to capture the essential features of the experiment. Complete phase diagrams for a recurrent neural network of binary units with asynchronous dynamics, which only exhibit fixed-point solutions, were obtained for both finite and extensive loading of patterns [12]. An approximate solution for the transient dynamics was also derived recently by means 
of the dynamical replica theory [13], and the coexistence of several attractors including correlated states were obtained for both finite and infinite loading. However, the models for SSP are restricted to a dominating Hebbian interaction in which $1 \leq J_{H} / J_{S}<\infty$ leading only to fixed-point solutions. The case of network models with dominating SSP has not been analyzed and it is interesting to find out what kind of solutions appear in this case.

The purpose of this work is to present a summary of results for the competition between pattern reconstruction of a Hebbian type and SSP in the full range of the ratio $J_{H} / J_{S}$, in particular for dominating SSP, which is expected to lead to cyclic behavior. The dynamics of a feed-forward layered network model of binary units is used [14] and the results are compared with those for ASP. How the model is solved, the relation to other models and a full discussion of results for SSP can be found elsewhere [15]. A dynamical procedure must be used since the synaptic interactions are asymmetric due to the feed-forward nature of the network. The model is expected to exhibit some of the relevant features of more realistic models.

It will be shown that a new phase of stationary cyclic solutions with period two is obtained, independently of the number of condensed patterns $c$ with macroscopic overlap with the states of the network, for sufficiently weak Hebbian interaction, and that the stability of this phase is strongly dependent on $c$. We focus mainly on the differences between this model and that for ASP competing with pattern reconstruction in a feedforward layered network [1], where the cycles have period $c$, for arbitrary $c$, and the stability of these solutions does not depend on this parameter. Phase diagrams and the behavior of the overlaps in the stationary cyclic phase are shown here and the effects of stochastic noise are briefly discussed.

The paper is organized as follows. First we present the model and the recursion relations for the relevant parameters. Then we show some of the main results for the overlaps and the phase diagrams and conclude with a further discussion and an outlook.

\section{THE MODEL}

The model is an Ising spin neural network composed of $L$ layers, each containing $N$ spins $\sigma_{i}(l) \in\{1,-1\}$, where $i=1, \ldots, N$ represents the unit and $l=1, \ldots, L$ the layer. If neuron $i$ on layer $l$ is firing or at rest, $\sigma_{i}(l)= \pm 1$, respectively. The state of unit $i$ on layer $l+1$ is determined in parallel by the collective state $\vec{\sigma}(l)=\left\{\sigma_{1}(l), \ldots, \sigma_{N}(l)\right\}$ of the previous layer according to the stochastic rule with conditional probability

$$
\begin{aligned}
\mathrm{P}\left(\sigma_{i}(l+1) \mid \vec{\sigma}(l)\right) & =\frac{\exp \left[\beta \sigma_{i}(l+1) h_{i}(l+1)\right]}{\left.2 \cosh \left[\beta h_{i}(l+1)\right)\right]}, \\
h_{i}(l+1) & =\sum_{j=1}^{N} J_{i j}(l) \sigma_{j}(l)
\end{aligned}
$$

where $h_{i}(l+1)$ is the local field produced by the entire layer $l$ on neuron $i$ of layer $l+1$ and $J_{i j}(l)$ represents the strength of the connection between unit $j$ on layer $l$ to unit $i$ on layer $l+1$. The parameter $\beta=T^{-1}$ controls the synaptic noise such that the dynamics is fully deterministic when $T \rightarrow 0$ and fully random when $T \rightarrow \infty$. In the former case the 
dynamics of a neuron assumes the form $\sigma_{i}(l+1)=\operatorname{sgn}\left[h_{i}(l)\right]$. There is no feedback in the updating of the units and all units on each layer are updated simultaneously. Thus, the layer index may be thought as a discrete time step, and the network evolves according to a parallel dynamics.

A macroscopic set of $p=\alpha N$ statistically independent and identically distributed random patterns $\left\{\xi^{\mu}(l)\right\}, \mu=1, \ldots, p$, with components $\xi_{i}^{\mu}(l)= \pm 1$ and probability $\frac{1}{2}$ for either value, are stored on every layer independently of other layers according to a learning rule in two stages involving patterns on two consecutive layers. There is a stage of Hebbian learning as a static process reinforcing the same pattern on every layer, and a second stage in which the patterns are presented to the network in sequential order, with a given pattern $\mu$ on one layer associated with patterns $\mu+1$ and $\mu-1$ on the next layer. The second stage may be considered as a dynamic process favoring transitions between consecutive patterns. The synaptic interactions between neurons are then given by

$$
J_{i j}(l)=\frac{1}{N} \sum_{\mu, \rho=1}^{p} \xi_{i}^{\mu}(l+1) X_{\mu \rho} \xi_{j}^{\rho}(l),
$$

in which $X_{\mu \rho}$ are the elements of the matrix

$$
\mathbf{X}=\left(\begin{array}{cc}
\mathbf{A} & 0 \\
0 & \mathbf{B}
\end{array}\right)
$$

and

$$
\begin{aligned}
& A_{\mu \rho}=v \delta_{\mu, \rho}+(1-v)\left(\delta_{\mu, \rho+1}+\delta_{\mu, \rho-1}\right), \\
& B_{\mu \rho}=b \delta_{\mu, \rho}+(1-b)\left(\delta_{\mu, \rho+1}+\delta_{\mu, \rho-1}\right) .
\end{aligned}
$$

The matrices $\mathbf{A}$ and $\mathbf{B}$ have dimensions $c \times c$ and $(p-c) \times(p-c)$, respectively. The diagonal two-block interaction matrix reflects the fact that the patterns are grouped into two independent cycles, one for the condensed patterns $\left(\vec{\xi}^{c+1}(l)=\vec{\xi}^{1}(l)\right)$ and the other one for the non-condensed patterns $\left(\vec{\xi}^{p+1}(l)=\vec{\xi}^{c+1}(l)\right)$. Since the off-diagonal blocks are absent in our model, there is no connection between condensed and non-condensed patterns and this feature guarantees the applicability of the signal-to-noise analysis [14], in which the local field can be separated in a signal and a noise term. The choice of two independent parameters $v$ and $b(0 \leq v, b \leq 1)$ enables one to explore the effects of the relative weights of the interactions and the form of the noise on the dynamics of the network. When $b=1$ there is a purely Hebbian noise and when $b \neq 1$ there is a Hebbian plus sequential noise. In the case of ASP also discussed here, in which a pattern $\mu$ on a given layer is connected only to pattern $\mu+1$ on the following layer, $A_{\mu \rho}=v \delta_{\mu, \rho}+(1-v) \delta_{\mu, \rho+1}$ and $B_{\mu \rho}=b \delta_{\mu, \rho}+(1-b) \delta_{\mu, \rho+1}$. We restrict ourselves in the following to Hebbian noise and the general case for both forms of sequence processing can be found elsewhere $[1,15]$.

To describe the state of the network on a given layer $l$, we introduce the macroscopic overlap $m_{\mu}(l) \simeq O(1)$ between the configuration $\vec{\sigma}(l)$ and a condensed pattern $\mu$, as the large- $N$ limit of $m_{N}^{\mu}(l)=N^{-1} \sum_{i=1}^{N} \xi_{i}^{\mu}(l)\left\langle\sigma_{i}(l)\right\rangle$, in which $\mu=1, \ldots, c$ and the brackets $\langle\ldots\rangle$ represent a thermal average with Eq. (1). The self-averaging property may be used 
to write $m_{\mu}(l)=\left\langle\left\langle\xi_{i}^{\mu}(l) \sigma_{i}(l)\right\rangle\right\rangle_{\vec{\xi}}$ in the limit $N \rightarrow \infty$, since the number of condensed patterns is finite. Here $\langle\ldots\rangle_{\vec{\xi}}$ denotes an explicit configurational average over the condensed patterns. The remaining $p-c$ non-condensed patterns have microscopic overlaps $M_{N}^{\mu}(l) \simeq O(1 / \sqrt{N})$. The solution of the model consists in obtaining a dynamical equation for the overlaps that allows to predict the macroscopic state on any layer $l$ for a given state on the first layer. The local field on layer can be written [14], in the large- $N$ limit, as a sum of a signal and a noise term due to the macroscopic and microscopic overlaps on the previous layer, respectively. The noise follows a Gaussian distribution with mean zero and a layer-dependent variance $\Delta(l)$. We refer the reader to a recent work for a further discussion and a detailed derivation of all recursion relations [15].

We restrict ourselves here to the discrete dynamical equation for the macroscopic vector overlap $\vec{m}(l)=\left(m_{1}(l), \ldots, m_{c}(l)\right)$

$$
\vec{m}(l+1)=\left\langle\vec{\xi} \int D z \tanh \{\boldsymbol{\beta}[\vec{\xi} \cdot \mathbf{A} \vec{m}(l)+\Delta(l) z]\}\right\rangle_{\vec{\xi}},
$$

where $D z=e^{-z^{2} / 2} d z / \sqrt{2 \pi}$. The spin-glass order parameter $q(l)=\left\langle\langle S(l)\rangle^{2}\right\rangle_{\vec{\xi}}$, which enters in the recursion relation for $\Delta(l)$,

$$
\Delta^{2}(l+1)=\alpha+(1-q(l))^{2} \beta^{2} \Delta^{2}(l)
$$

is given by

$$
q(l)=\left\langle\int D z \tanh ^{2}\{\beta[\vec{\xi} \cdot \mathbf{A} \vec{m}(l)+\Delta(l) z]\}\right\rangle_{\vec{\xi}} .
$$

This is the complete set of equations to be solved in the case of Hebbian noise and the situation is more complicated in the case of full noise $(b \neq 1)[1,15]$. The transient dynamics and the stationary states of the network model can be studied in full detail and we discuss here only the latter.

\section{RESULTS}

The results are shown mainly in the form of phase diagrams in which each phase represents a different behavior of the network achieved by iterating numerically the system of recursion relations until the network reaches a stationary solution. Nonstationary states could also appear since the synaptic matrix $J_{i j}(l)$ is always asymmetric. The Hopfield ansatz $m_{\mu}(1)=\delta_{\mu, 1}(\mu=1, \ldots, c)$ is used as an initial condition in all the cases studied.

First we consider the solutions for finite loading, where $\alpha=0$, in order to discuss the phases that appear, and in that case we have the fixed-point value $\Delta^{*}(l)=0$. In Figs. 1(a) and $1(\mathrm{~b})$ we show the $(v, T)$ phase diagrams for the symmetric and asymmetric cases, respectively. There is a paramagnetic phase (P) where $\vec{m}=0$ and $q=0$ above a line of continuous bifurcation from a phase of symmetric-like fixed-point solutions (S) which have equal or nearly equal overlap components. The Hopfield-like fixed-point solutions (H) have one large condensed overlap component and the other ones are either small or zero. These fixed-point states are qualitatively the same for both the symmetric and 

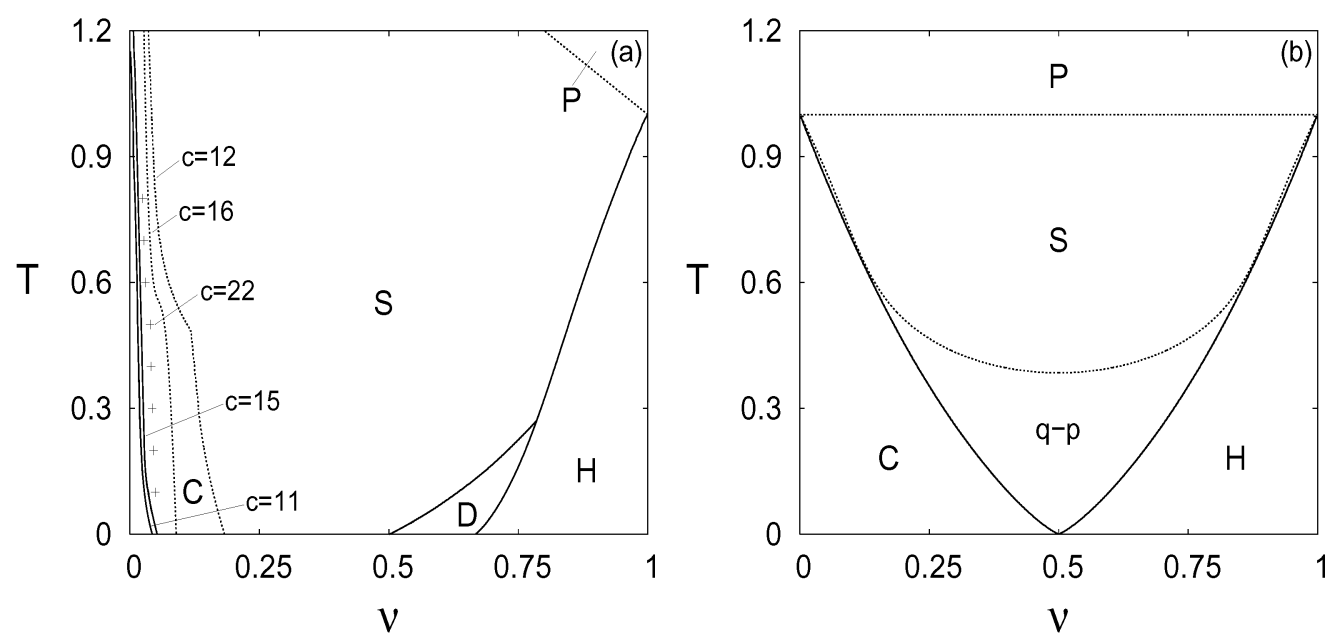

FIGURE 1. Phase diagrams for (a) SSP and (b) ASP, both for purely Hebbian noise and finite loading $(\alpha=0)$. The dotted and full lines indicate, continuous and discontinuous transitions, respectively. The phases are described in the text. The unlabeled phase boundaries are independent of $c$.

the asymmetric case. The phase boundaries for $v>0.5$ practically do not depend on $c$ although they are different for SSP and ASP. That is also the case for ASP when $v<0.5$, in distinction to the dependence on $c$ for SSP shown in Fig. 1(a) that will be discussed below in more detail. In the case of ASP only the boundary between the S phase and the phase of quasi-periodic states (q-p) depends on $c$ and it is shown in Fig. 1(b) only for $c=4$. In addition, there is a $v \Leftrightarrow(1-v)$ duality between phases, $H \Leftrightarrow C$ in the case of ASP, where $C$ is the phase of cyclic solutions, which relates the solutions for $v>0.5$ with the solutions for $v<0.5$, and this follows from the symmetry properties of the matrix A [4]. There is no duality, instead, in the case of SSP.

The first difference between the two cases appears when $v \approx 0.5$. At high $T$ the stabilizing effect of the static process (Hebbian term) locks the transition between the patterns and leads to symmetric states. At low $T$ the Hebbian term fails to lock the transitions and we have different phases in the two cases. In the SSP we have a phase of correlated fixed-point attractors (D) in which the stationary vector overlap has the form $\vec{m}=\left(1 / 2^{7}\right)(0,0,1,3,13,51,77,51,13,3,1,0,0)$, for $c=13$ when $T \rightarrow 0$, where the central component is the stimulated one. An increase in the synaptic noise $T$ only affects the values of each component, with the qualitative form of the vector remaining the same. The correlation coefficients between these vector overlaps decay to zero as the distance with the initially stimulated patterns increases.

We consider now the phase of stationary cyclic solutions (C) for SSP. The appearance of cycles for $v<0.5$ is a remarkable property in SSP, which has not been discussed before. Fig. 2(a) illustrates the dependence of the first seven components of the vector overlap with respect to time $t$, again for $c=13$ condensed patterns, when the network reaches a stationary cyclic solution after a transient time. The oscillating vector overlap 

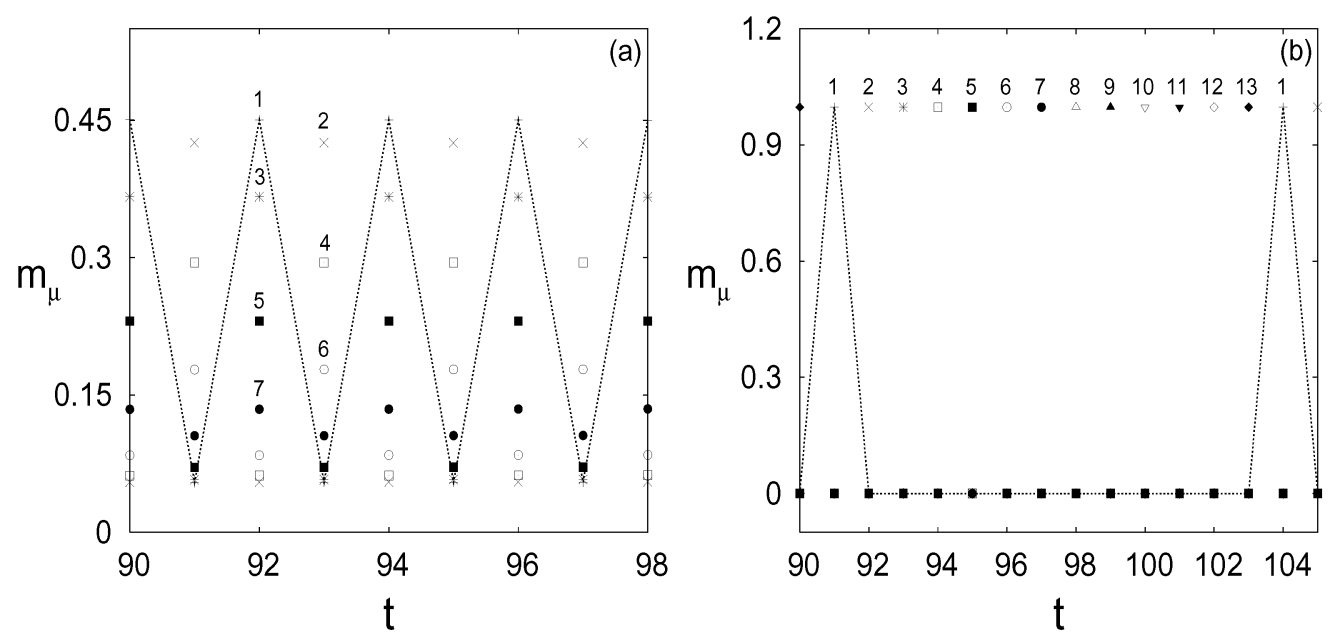

FIGURE 2. Overlap components as functions of discrete time $t$ (or layer $l$ ) for (a) SSP and (b) ASP for a typical point inside the cyclic phase $\mathrm{C}$. The values of parameters are $c=13, \alpha=0, T=0.3, v=0.01$ and Hebbian noise, for both figures. Each kind of point represents a different overlap component and is marked by a label. The dotted lines are a guide to the eye for the dynamics of the first overlap component. The dynamics of the other overlap components follows in a similar way.

$\vec{m}(t+2)=\vec{m}(t)$ has period two, with each overlap component assuming a larger and a smaller value at each time step, with a decreasing oscillation amplitude as we move away from the stimulated pattern $(\mu=1)$. The overlap components in the cyclic phase have the symmetry $m_{\mu+n}(t)=m_{\mu-n}$, where $\mu$ is the stimulated pattern and $n=1, \ldots,(c-1) / 2$ for odd $c$ and $n=1, \ldots,(c-2) / 2$ for even $c$. In the case of even $c$ we have this same kind of cyclic solutions and another one in which all the overlap components keep oscillating between the same two values at each time step. The main feature of these cyclic states is that they have always period two, independently of the number of condensed patterns c. Coming back to Fig. 1(a), we notice that the stability of the cyclic phase has a strong dependence on $c$, with its size decreasing or increasing with an increase of $c$, if $c$ is even or odd, respectively. The transition between the $\mathrm{S}$ and $\mathrm{C}$ phases also depends on $c$, being continuous or discontinuous if $c$ is even or odd, respectively.

In Fig. 2(b) we show the overlap components as functions of time $t$, after a transient, when the network reaches the stationary cyclic solution in the case of ASP. One can see that one overlap component is one and all the others are zero at each time step. This fact characterizes the retrieval of a sequence of stored patterns in which the network makes a transition from one pattern to the next at each time step. In contrast to SSP, these cyclic solutions have period $c(c=13$ in Fig. 2(b)), and this is also the case for even $c$. Moreover, the transition between the $\mathrm{S}$ and $\mathrm{C}$ phases is always discontinuous and the phase boundary practically does not depend on $c$, which reflects the independence of the stability of these solutions with respect to this parameter.

Finally, we consider the effects of stochastic noise due to a macroscopic number of stored patterns $p=\alpha N$ on the phase diagram, and we present the results in Fig. 3 only 


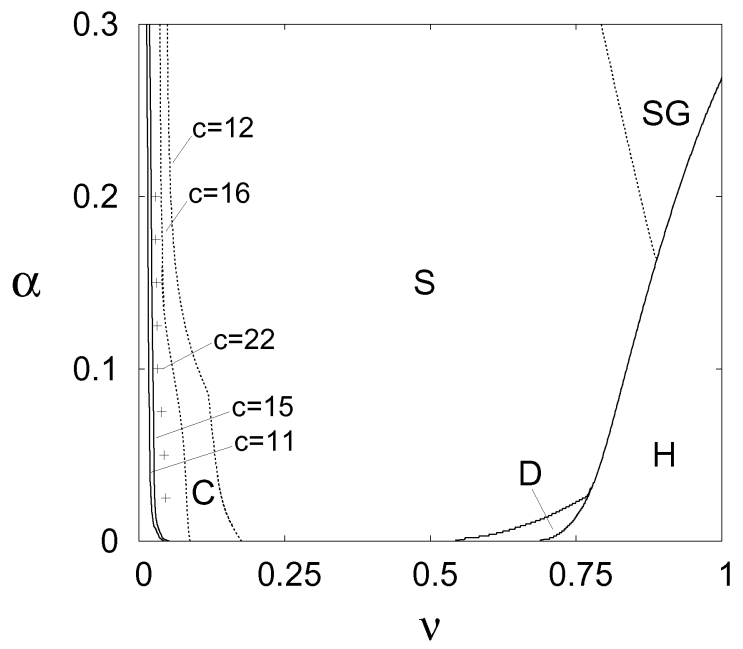

FIGURE 3. Phase diagram for SSP at $T=0$ and a purely Hebbian noise $(b=1)$. The dotted and full lines indicate, respectively, continuous and discontinuous transitions. The phases are described in the text.

for SSP, again for a purely Hebbian noise at $T=0$. For the variance of the noise we choose the initial condition $\Delta^{2}(1)=\alpha$. As usual in the layered network, there is now a spin-glass phase (SG) with $\vec{m}=0$ and $q \neq 0$. The other phases are qualitatively similar to those described previously and the cyclic phase also preserves the same properties as in the finite loading regime. The cyclic solutions are again of period two, independently of $c$, and the phase boundary between the $\mathrm{C}$ and $\mathrm{S}$ phases depends strongly on $c$. The continuity of this transition and the qualitative form of the cyclic solutions depends on the parity of $c$ in the same way as for the $\alpha=0$ case. Again, the boundaries between phases of fixed-point solutions are fairly independent of $c$ and the phase diagram for $v>0.5$ is similar to that of a recurrent network in equilibrium [12]. For $v=1$ we recover the critical storage ratio $\alpha_{c} \simeq 0.269$ for the Hebbian layered network model [14]. For the effects of stochastic noise in ASP we refer the reader to [1] for a detailed discussion.

\section{CONCLUSIONS AND OUTLOOK}

We presented here the dynamics of competition between pattern reconstruction and sequence processing in an exactly solvable feed-forward layered neural network model of binary units and patterns for both finite and extensive loading. Two kinds of sequence processing were discussed: SSP where a pattern $\mu$ is connected to patterns $\mu+1$ and $\mu-1$ on the next layer and ASP where a pattern $\mu$ is only connected to pattern $\mu+1$ on the next layer. In both cases there is a Gaussian noise due to the non-condensed patterns in the local field and the model can be solved for finite or extensive loading of patterns. The extension to $Q$-state $(Q \geq 3)$ neurons and patterns as well as continuous neurons 
is straightforward. Non-stationary quasi-periodic states were found in the case of ASP $[4,1]$ which apparently are absent in SSP.

There are important differences in the phase of cyclic solutions, as discussed here, and these are phases which appear for $v \leq 0.5$ for either SSP or ASP. To summarize, in the case of SSP the period of cycles is always two, independently of the number of condensed patterns, but the stability of the cyclic phase is strongly dependent on $c$. On the other hand, in ASP there are cycles of period $c$ while the stability of the phase practically does not depend on $c$. Despite the fact that the synaptic matrix $J_{i j}(l)$ is always asymmetric due to the dependence of the patterns with respect to the layer index (the feed-forward nature of the network), what seems to determine these differences is the symmetry of $\mathbf{A}$ with respect to the pattern indexes.

Since the phase diagrams for $v>0.5$ in SSP are qualitatively similar to those for a recurrent network in equilibrium, one may also expect that cycles of period two appear in SSP in a recurrent network with parallel dynamics, for a sufficiently weak Hebbian interaction. Work along this line, currently in progress, indicates that this is indeed the case [16].

$>$ From an experimental point of view, there is the possibility of making qualitative extended predictions for the kind of Miyashita et al. experiments [8,9], which revealed that temporal correlations expressed by the position number of the patterns in a sequence during learning are converted into spatial correlations of the stored activity patterns in the cortex of monkeys. This was interpreted as the presence of correlated states in a recurrent network [11]. Also, it became apparent from this kind of experiments that there is a direct connection between persistent (delay) activity and fixed-point attractor dynamics. It would be interesting to see if this connection extends to cyclic attractor dynamics and both further experimental and theoretical work has to be done in order to settle this issue.

One may speculate that if the training of the random patterns, which seems to be a realization of a Hebbian rule, is not quite successful, one may have a situation as that described here for small $v$ with the presence of cyclic states of period two. For a possible quantitative prediction of experiments one has to use, of course, a more appropriate model in terms of integrate-and-fire neurons rather than the binary model discussed in this work.

\section{ACKNOWLEDGMENTS}

The work of one of the authors (WKT) was financially supported, in part, by CNPq (Conselho Nacional de Desenvolvimento Científico e Tecnológico), Brazil. Grants from CNPq and FAPERGS (Fundação de Amparo à Pesquisa do Estado de Rio Grande do Sul), Brazil, to the same author are gratefully acknowledged. F. L. Metz acknowledges a fellowship from CNPq.

\section{REFERENCES}

1. F. L. Metz, and W. K. Theumann, Phys. Rev. E 72, 021908 (2005).

2. J. J. Hopfield, Proc. Natl. Acad. Sci. USA 79, 2554-2558 (1982). 
3. H. Sompolinsky, and I. Kanter, Phys. Rev. Lett. 57, 2861-2864 (1986).

4. A. C. C. Coolen, and D. Sherrington, J. Phys. A: Math. Gen. 25, 5493-5526 (1992).

5. W. Whyte, D. Sherrington, and A. C. C. Coolen, J. Phys. A: Math. Gen. 28, 3421-3437 (1995).

6. A. Düring, A. C. C. Coolen, and D. Sherrington, J. Phys. A: Math. Gen. 31, 8607-8621 (1998).

7. M. Kawamura and M. Okada, J. Phys. A: Math. Gen. 35, 253-266 (2002).

8. Y. Miyashita, and H. S. Chang, Nature 331, 68-70 (1988).

9. Y. Miyashita, Nature 335, 817-820 (1988).

10. G. Mongillo, D. J. Amit and N. Brunel, Eur. J. Neurosci. 18, 2011-2024 (2003), for a recent review.

11. M. Griniasty, M. V. Tsodyks, and D. J. Amit, Neural Comp. 5, 1-17 (1993).

12. L. F. Cugliandolo, and M. V. Tsodyks, J. Phys. A: Math. Gen. 27, 741-756 (1994).

13. T. Uezu, A. Hirano, and M. Okada, J. Phys. Soc. Japan 73, 867-874 (2004).

14. E. Domany, W. Kinzel, and R. Meir, J. Phys. A: Math. Gen. 22, 2081-2102 (1989).

15. F. L. Metz, and W. K. Theumann, submitted to Phys. Rev. E.

16. F. L. Metz, and W. K. Theumann, unpublished. 
Copyright of AIP Conference Proceedings is the property of American Institute of Physics and its content may not be copied or emailed to multiple sites or posted to a listserv without the copyright holder's express written permission. However, users may print, download, or email articles for individual use. 